Mechanisms governing the global regulation of mycotoxin production and pathogenicity by Penicillium expansum in postharvest fruits

Keller, N.P. University of Wisconsin-Madison

Sionov, E. Agricultural Research Organization

Barad-Kotler, S. Agricultural Research Organization

Project award year: 2017

Three year research project 
26.11.2020

Final Report for BARD-5042

\section{Mechanisms governing the global regulation of mycotoxin production and pathogenicity by Penicillium expansum in postharvest fruits}

\section{Abstract}

The original objectives of the study, as defined in the approved proposal, are:

1. To characterize the relationship of CreA and LaeA in regulation of PAT production

2. To understand how PacC modulates $P$. expansum pathogenicity on apples

3. To examine if other secondary metabolites are involved in virulence or $P$. expansum fitness 4. To identify the signaling pathways leading to PAT synthesis

Penicillium expansum, the causal agent of blue mould rot, is a critical health concern because of the production of the mycotoxin patulin (PAT) in colonized apple fruit tissue. Although PAT is produced by many Penicillium species, the factors activating its biosynthesis were not clear. This research focused on host and fungal mechanisms of activation of LaeA (the global regulator of secondary metabolism), PacC (the global $\mathrm{pH}$ modulator) and CreA (the global carbon catabolite regulator) on PAT synthesis with intention to establish $P$. expansum as the model system for understanding mycotoxin synthesis in fruits. The overall goal of this proposal is to identify critical host and pathogen factors that mechanistically modulate $P$. expansum genes and pathways to control activation of PAT production and virulence in host.

Several fungal factors have been correlated with disease development in apples, including the production of PAT, acidification of apple tissue by the fungus, sugar content and the global regulator of secondary metabolism and development, LaeA. An increase in sucrose molarity in the culture medium from 15 to $175 \mathrm{mM}$ negatively regulated laeA expression and PAT accumulation, but, conversely, increased creA expression, leading to the hypothesis that CreA could be involved in $P$. expansum PAT biosynthesis and virulence, possibly through the negative regulation of LaeA. We found evidence for CreA transcriptional regulation of laeA, but this was not correlated with PAT production either in vitro or in vivo, thus suggesting that CreA regulation of PAT is independent of LaeA. Our finding that sucrose, a key ingredient of apple fruit, regulates PAT synthesis, probably through suppression of laeA expression, suggests a potential interaction between CreA and LaeA, which may offer control therapies for future study. We have also identified that in addition to PAT gene cluster, CreA regulates other secondary metabolite clusters, including citrinin, andrastin, roquefortine and communesins, during pathogenesis or during normal fungal growth.

Following creation of $P$. expansum pacC knockout strain, we investigated the involvement of the global $\mathrm{pH}$ regulator $\mathrm{PacC}$ in fungal pathogenicity. We demonstrated that disruption of the $\mathrm{pH}$ signaling transcription factor PacC significantly decreased the virulence of $P$. expansum on deciduous fruits. This phenotype is associated with an impairment in fungal growth, decreased accumulation of gluconic acid and reduced synthesis of pectolytic enzymes. We showed that glucose oxidaseencoding gene, which is essential for gluconic acid production and acidification during fruit colonization, was significantly down regulated in the $\triangle P$ PepacC mutant, suggesting that gox is PacCresponsive gene. We have provided evidence that deletion of gox gene in $P$. expansum led to a reduction in virulence toward apple fruits, further indicating that GOX is a virulence factor of $P$. expansum, and its expression is regulated by PacC. It is also clear from the present data that PacC in $P$. expansum is a key factor for the biosynthesis of secondary metabolites, such as PAT.

On the basis of RNA-sequencing (RNA-seq) analysis and physiological experimentation, the $P$. expansum $\triangle l a e A, \triangle c r e A$ and $\triangle p a c C$ mutants were unable to successfully colonize apples for a multitude of potential mechanisms including, on the pathogen side, a decreased ability to produce proteolytic enzymes and to acidify the environment and impaired carbon/nitrogen metabolism and, on the host side, an increase in the oxidative defence pathways. Our study defines these global regulatory factors and their downstream signalling pathways as promising targets for the development of strategies to fight against this post-harvest pathogen. 
Summary Sheet

\section{Publication Summary}

\begin{tabular}{|c|c|c|c|}
\hline PubType & IS only & Joint & US only \\
\hline Reviewed & 0 & 2 & 0 \\
\hline
\end{tabular}

\section{Training Summary}

\begin{tabular}{|c|c|c|c|c|}
\hline Trainee Type & Last Name & First Name & Institution & Country \\
\hline Postdoctoral Fellow & Kumar & Dilip & ARO & Israel \\
\hline Postdoctoral Fellow & Barda & Omer & ARO & Israel \\
\hline Postdoctoral Fellow & Tannous & Joanna & University of Wisconsin- Madison & USA \\
\hline Ph.D. Student & Luciano-Rosario & Dianiris & University of Wisconsin- Madison & USA \\
\hline Ph.D. Student & Eagen & Justin & University of Wisconsin-Madison & USA \\
\hline
\end{tabular}


26.11.2020

Final Report for BARD-5042

\section{Contribution of the collaboration}

All project objectives were promoted by the Israeli and USA labs that share equal responsibility for guiding an integrated research effort to study the mechanisms of the global regulation of mycotoxin production and virulence of Penicillium expansum on apples. The investigators also collaborated on publications and vouch for the accuracy and quality of the research in its entirety. Our collaboration has been characterized by a spirit of equality and focus on the scientific goals. We have established a policy of joint authorship; we have published two publications together during the project. Additionally, the labs share materials and Keller lab has sent the Sionov lab a strain of $P$. expansum that is easier to transform. The two labs also have joint laboratory meetings via SKYPE and meet for strategizing goals at international fungal genetics meetings. 
26.11.2020

Final Report for BARD-5042

\section{Significance of main scientific achievements}

During the first year of the project we characterized the mechanism of activation of the LaeA, the global regulator of mycotoxin production, in patulin (PAT) synthesis. We examined a series of intrinsic critical host factors that change during fruit ripening - including sugars, organic acids, $\mathrm{pH}$, and phenols - and present their impact on PAT synthesis and laeA expression. Intrinsic apple factors differentially contribute to the complex activation of the PAT gene cluster and contribute to the accumulation of PAT in colonized fruits (Kumar et al., 2018). The findings provide an initial insight into fruit intrinsic factors that may have a significant impact on $P$. expansum's capability to produce and accumulate PAT in apple. The correlation of increased sucrose and PAT production in apples was not observed in growth media suggesting that other metabolites in the host have a large impact on PAT synthesis by the fungus. We show here that combinations of apple nutrients can alter the impact of sucrose on laeA and pat expression as well as PAT production in vitro and suggest a similar but even more complex affect occurs in fruit (Kumar et al., 2018).

To assess the relative contributions of CreA to LaeA regulation of PAT production, we created creA knock-out strain. First we created a $4 k u 70$ strain of $P$. expansum to increase homologous recombination events, as described for other fungi. To assess any functional role of the $P$. expansum transcription factor CreA in the physiology and virulence of this pathogenic fungus, we deleted creA in $\Delta k u 70$, obtaining several correct deletion mutants. The ability of the $P$. expansum strains to produce PAT was assessed previously under 50 and $175 \mathrm{mM}$ sucrose concentrations, one of the primary sugars present in apples. As observed previously in P. expansum Pe-21, we also found a negative correlation between PAT accumulation and creA expression in the WT and PhleoR (strain containing the phleomycin-resistance cassette inserted at a different site in $P$. expansum genome) control strains with increasing sucrose content. Although fungal growth in the presence of $50 \mathrm{mM}$ sucrose resulted in patulin production of $300 \mu \mathrm{g} / \mathrm{g}$ DW (dry weight) in both control strains, the level of patulin declined to $70 \mu \mathrm{g} / \mathrm{g}$ DW in the presence of $175 \mathrm{mM}$ sucrose. In contrast, the relative expression of $c r e A$ showed a 2.2 increase in the presence of $175 \mathrm{mM}$ relative to $50 \mathrm{mM}$ sucrose. We were also interested in assessing laeA levels, as we hypothesized that deletion of creA will result in increased laeA expression in high sugar environments with resultant higher PAT production. Our results partially supported this hypothesis. laeA expression increased in both sucrose regimes in the $\Delta c r e A$ strains relative to the control strains; there was no PAT production in the $\Delta c r e A$ strains under both sucrose regimes. These results suggest a LaeA-independent role for CreA in PAT synthesis. 
Next, following characterization the mechanism of activation of the LaeA, the global regulator of mycotoxin production, in PAT synthesis and indication that LaeA is independent of the global carbon catabolite regulator, CreA, in PAT synthesis we assessed virulence of the creA mutants on apple to see if this global transcription factor impacts pathogenicity. Colonization of Golden Delicious apples by $\triangle c r e A$ strains showed a significant reduction in the rotten colonized area relative to that of the WT strain. The analysis of PAT accumulation in the inoculated tissue 7 days after inoculation revealed no PAT synthesis by the $\Delta c r e A$ strains, compared with both WT and PhleoR control strains. To gain an additional understanding of the potential mechanism underlying the reduced pathogenicity of the $\triangle c r e A$ strains, the mutants were assessed for several physiological attributes that have previously been linked with virulence in this pathogen. A proposed virulence mechanism of P. expansum is its ability to reduce the $\mathrm{pH}$ of infected apple tissue through the production of gluconic acid (GLA). Here, the growth of both WT and PhleoR control strains in the presence of a high sucrose level at an initial $\mathrm{pH}$ 7 led to a reduction of almost $3 \mathrm{pH}$ units after $24 \mathrm{~h}$ and, in the following $48 \mathrm{~h}$, the $\mathrm{pH}$ remained between $\mathrm{pH} 4.5$ and $\mathrm{pH}$ 5.0. This acidification of the medium was accompanied by increased accumulation of GLA. In contrast, the $\triangle c r e A$ strains showed no acidification of the medium and no GLA accumulation at any time point. Given that GLA formation requires glucose oxidase activity, the lack of gox2 (glucose oxidase gene) expression in the $\Delta c r e A$ strains explains the absence of GLA formation (Tannous et al., 2018).

For assessment of involvement of the $\mathrm{pH}$ global regulator PacC in pathogenicity of $P$. expansum we created pacC knock-out in P. expansum Pe-21 strain. Physiological analysis showed that $\Delta$ pacC mutant was reduced in fungal growth and delayed in spore formation and germination compared with control strain. Virulence of WT and $\triangle P e p a c C$ strains was evaluated on apple fruits, which are the most important hosts of $P$. expansum. $\triangle$ PepacC mutant was near avirulent in inoculated fruits compared to those inoculated with WT strain. Deletion of PepacC dramatically reduced disease incidences in apple fruits (5 days after inoculation). These results indicated that PePacC plays important roles in pathogenicity in P. expansum. We also compared PAT production of WT and $\triangle P$ epacC in different $\mathrm{pH}$ conditions. It was found that deletion of PepacC had strikingly negative effects on PAT production under both acidic and alkaline conditions. These results indicated that PePacC is an important regulator in PAT biosynthesis of $P$. expansum in both acidic and alkaline conditions. Similarly to $c r e A$ gene, disruption of pac $C$ resulted in reduced pathogenicity of $P$. expansum in apple fruits through mediating a virulence factor glucose oxidase (GOX2). Our results demonstrated that pacC mutants reduced gluconic acid (which is regulated by gox expression) and PAT accumulation in apples and showed reduction in fungal pathogenicity, compared to the WT.

We have also identified that in addition to PAT gene cluster, other secondary metabolite clusters are regulated by global transcription factors during pathogenesis or during normal fungal growth. The 
global carbon catabolite regulator, CreA, affects the production of other known secondary metabolites, including citrinin, andrastin, roquefortine and communesins. In addition to its negative regulation of PAT synthesis, creA loss decreases the production of citrinin. Because citrinin has been reported occasionally as a contaminant of apples, we also assessed infected apples for this mycotoxin, but could not detect it in either the PhleoR control strain or the $\Delta c r e A$ mutant. Citrinin biosynthetic gene cluster showed an increase in expression in the $\Delta c r e A$ mutant, however, no mycotoxin accumulation was produced in apple infected by the mutant. It remains unclear whether the nondetection of citrinin by the control strain in apples is caused by a deficiency in production or stability inside apple tissues or a lack of efficient and reliable recovery and detection. Some known secondary metabolites, andrastin $\mathrm{A}$, roquefortine $\mathrm{C}$ and communesin $\mathrm{D}$, were produced to slightly different, but significant, levels in the creA deletion strain, although this was not reflected in the transcript levels of the synthase genes required for these metabolites, possibly because of the relatively minor changes in production of these metabolites.

In order to identify signaling pathways that are modulated by both CreA and LaeA, we sent RNA samples of the $P$. expansum WT, $\triangle l a e A, \Delta c r e A$ and $\triangle p a c C$ strains, isolated following inoculation from apple fruits, for RNA-Seq using next generation large-scale genome-sequencing technology the Illumina high throughput sequencing platform. On the basis of RNA-sequencing (RNA-seq) analysis and physiological experimentation, these mutants were unable to successfully colonize apples for a multitude of potential mechanisms including, on the pathogen side, a decreased ability to produce proteolytic enzymes and to acidify the environment and impaired carbon/nitrogen metabolism and, on the host side, an increase in the oxidative defence pathways. Transcript analysis of $P$. expansum infection of apples showed that the global transcription factors LaeA, PacC and CreA regulate several fungal processes potentially involved in the reduced pathogenicity of the mutant strains. Our study unveiled the mechanism of activation of transcription pathways regulating the toxic secondary metabolite accumulation at the infection site, and their effect on pathogenicity processes in postharvest diseases caused by Penicillium. We described at the molecular level the mechanism by which pathogenicity and the accumulation of PAT by P. expansum regulate the fruit colonization during their postharvest life. The use of plant systems to investigate the molecular mechanism of PAT pathogenicity may offer insight into the mode of action in animal tissue. The results of the research described in this study will facilitate the development of non-chemical approaches to the reduction of postharvest diseases that occur during storage. Thus, such knowledge will be widely applicable to many economically important postharvest diseases, and will assist to develop rational approaches for management of fungal infections of fresh fruit accompanied by mycotoxin contamination. 
Publications for Project IS-5042-17C

\begin{tabular}{|c|c|c|c|c|c|c|}
\hline $\begin{array}{c}\text { Stat } \\
\text { us }\end{array}$ & Type & Authors & Title & Journal & $\begin{array}{l}\text { Vol:pg } \\
\text { Year }\end{array}$ & $\begin{array}{c}\text { Cou } \\
\text { n }\end{array}$ \\
\hline Published & Reviewed & $\begin{array}{l}\text { Joanna Tannous, } \\
\text { Dilip Kumar, Noa } \\
\text { Sela, Edward } \\
\text { Sionov, Dov Prusky, } \\
\text { Nancy P. Keller }\end{array}$ & $\begin{array}{c}\text { Fungal attack and host defence } \\
\text { pathways unveiled in } \\
\text { near?avirulent interactions of } \\
\text { Penicillium expansum creA } \\
\text { mutants on apples }\end{array}$ & $\begin{array}{c}\text { Molecular Plant } \\
\text { Pathology }\end{array}$ & $\begin{array}{l}19: 2635- \\
26502018\end{array}$ & Joint \\
\hline Published & Reviewed & $\begin{array}{c}\text { Kumar D, Tannous } \\
\text { J, Sionov E, Keller } \\
\text { N, Prusky D. }\end{array}$ & $\begin{array}{l}\text { Apple Intrinsic Factors } \\
\text { Modulating the Global } \\
\text { Regulator, LaeA, the Patulin } \\
\text { Gene Cluster and Patulin } \\
\text { Accumulation During Fruit } \\
\text { Colonization by Penicillium } \\
\text { expansum }\end{array}$ & $\begin{array}{l}\text { Frontiers in Plant } \\
\text { Science }\end{array}$ & $\begin{array}{c}9: 1-13 \\
2018\end{array}$ & Joint \\
\hline
\end{tabular}


26.11.2020

Final Report for BARD-5042

\section{Appendix}

\section{Publications related to BARD-5042 project}

1. Tannous J, Kumar D, Barad S, Dubey A, Sionov E, Prusky D, Keller NP (2018). Fungal attack and host defense pathways unveiled in near avirulent interactions of Penicillium expansum creA mutants on apples. Mol. Plant Pathol. 19:2635-2650.

2. Kumar D, Tannous J, Keller NP, Sionov E, Prusky D (2018). Apple intrinsic factors modulating the global regulator, laeA, the patulin gene cluster and patulin accumulation during fruit colonization by Penicillium expansum. Front. Plant Sci. 9:1094.

3. Kumar D, Barad S, Chen Y, Luo X, Tannous J, Dubey A, Glam N, Li B, Keller N, Prusky D (2017). LaeA regulation of secondary metabolism and virulence in Penicillium expansum is mediated by sucrose. Mol. Plant. Pathol. 18:1150-1163.

4. Tannous J, Keller NP, Atoui A, Khoury AE, Lteif R, Oswald IP, Puel O (2018). Secondary metabolism in Penicillium expansum: Emphasis on recent advances in patulin research. Crit. Rev. Food Sci. Nutr. 58:12, 2082-2098, DOI: $10.1080 / 10408398.2017 .1305945$

5. Jurick WM, Peng H, Beard H, Garrett WM, Litchner FJ, Luciano-Rosario D, Macarisin O, Liu Y, Peter KA, Gaskins VL, Yang T, Mowery J, Bauchan G, Keller NP, Cooper B (2020). Blistering1 modulates Penicillium expansum virulence via vesicle-mediated protein secretion. Mol. Cell Proteomics. 19:344-361. doi:10.1074/mcp.RA119.001831.

6. Luciano-Rosario D, Keller NP, Wayne M. Jurick II WM (2020). Penicillium expansum: biology, omics, and management tools for a global postharvest pathogen causing blue mold of pome fruit. Mol. Plant Pathol. 11:1391-1404. 